\title{
Particle Tracing: Revisited
}

by M. Gharib ${ }^{\dagger}$ and C. Willert

Department of Applied Mechanics and Engineering Sciences

University of California, San Diego

La Jolla, California 92093-0310

\section{ABSTRACT}

Particle tracing is a simple technique used to infer global quantitative information regarding velocity and vorticity fields from flow images. In this paper, recent advances in solving major drawbacks associated with conventional particle tracing are discussed. A quantitative automatic scheme which eliminates the photographic step and is capable of constructing the velocity field directly from the experimental set up is presented.

\section{INTRODUCTION}

Flow visualization is a unique tool used to better understand complex flow structures. Usually, the nature of this understanding is qualitative, therefore a subsequent quantitative measurement is essential for further progress. Quantitative single point measurement techniques such as hot wire anamometry or laser doppler velocimetry are often used to obtain Eulerian velocity fields. These two techniques are typically limited to simultaneous sampling at a few spatial locations. In this respect, the global nature of the information contained in a single flow image invites one to develop quantitative methods to obtain multi-point spatial and temporal velocity or vorticity fields from such images.

Flow images produced by the pathlines of small distinct particles suspended in fluid are the best candidates for quantitative as well as qualitative flow observations. The patterns generated by the short pathlines are instantaneous and do not carry time history of past events which are often misleading in the continuous dye visualization techniques [1]. The velocity field can be obtained from trace photographs by measuring lengths and location of streaks. Modern history of fluid mechanics credits Ahlborne [2] and Prandtl [3] for development of the technique.

A major drawback in using particle tracing techniques has been the unacceptable amount of manual work which is required to obtain the velocity field from a large number of traces on each image. In recent years there have been renewed interests in making particle tracing techniques less laborious by incorporating image digitizers and computer image processing into the process of information extraction from the flow pictures. The degree of successfulness of these recent attempts should be judged based on the level of advancement that each method introduces to different steps of the particle tracing method. These steps include the type of particles, illumination techniques, photo or video graphic technique, digitization, trace recognition, sense of direction, error detection provisions, flow computation of randomly distributed data and overall reduction of processing time by automation of the different steps.

\footnotetext{
$\dagger$ Assistant Professor, Department of AMES, Member AIAA, APS, ASME

$\ddagger$ Graduate Researcher, Member of AIAA, ASME
}

In this paper, the parameters that are important in the practical use of the technique will be discussed, and the state of the art as well as some new ideas and methods that have been developed and implemented by the present authors and others will be reviewed.

\section{CRITERIA FOR THE PARTICLE SIZE}

The basic assumption in the particle tracing method is that the motion of the particles accurately represents the fluid motions. For photographic reasons, large particle sizes are desirable. However, large particle sizes and density mismatch between the particle and fluid might result in large differences between fluid and particle velocity.

For the case of the particle with density matching that of the fluid and small values of the particle Reynolds number, i.e.

$$
R_{p}=\frac{\left(U_{f}-U_{p}\right) D}{v}
$$

where $\mathrm{U}_{\mathrm{f}}$ and $\mathrm{U}_{\mathrm{p}}$ are the fluid and particle velocity respectively, and $\mathrm{D}$ is the particle diameter, Merzkirch [4] gives the particle response time to a step change in the fluid velocity as

$$
\tau_{p}=\frac{D^{2}}{18 v}
$$

Therefore for flows varying slower than $\tau_{\mathrm{p}}$, particles can accurately respond to the drag forces generated by the relative fluid motion. Large particles can also experience lift in flows with strong velocity shear. For particles smaller than the local Kolmogorov scale, this lift force is negligible (Saffman [5]). Agui and Jimenez [6] give the following relationship for the relative error of tracking for small density differences:

$$
\frac{\left|U_{f}-U_{p}\right|^{2}}{\left|U_{f}\right|^{2}}=\frac{r^{2} D^{2}}{10 \tau_{o p} v}
$$

where

$$
r=\frac{\rho_{p}}{\rho_{f}}-1
$$

( $\rho_{f}$ is the fluid density and $\rho_{p}$ is the particle density and $\tau_{o p}$ is a typical large eddy turn-over time.)

In general, it is important that particles be neutrally buoyant. However, since inertial and gravitational forces decrease rapidly with the particle size, particles with a size in the range of the local Kolmogorov scale should follow the flow correctly. 


\section{ILLUMINATION AND OPTICAL MODULATION OF THE PARTICLES}

The original experiments of Ahlborne and Prandtl, which involved free surface visualization, did not require sophisticated illumination methods. However, in order to visualize two components of the velocity in a three-dimensional flow field, illumination of a thin but finite cross-section of the flow is required. A sheet of light can be generated by passing a white light beam through a slit (Wiese Nielson [7], Imaichi and Ohmi [8]). The practical difficulties of collimating the light sheet for uniform thickness or generating a thin light sheet from an incoherent light source can be solved using lasers. Laser beams can be thinned, collimated and expanded to a sheet of light through the use of cylindrical lenses, mirrors or rapid light scanners. Dimotakis et.al. [9] pioneered the laser sheet method for particle tracing and obtained improved pictures due to the small depth of field in their images.

Since the laser sources have a narrow band of wave lengths, particles coated with fluorescent or phosphorescent materials can be selected to absorb and emit light at different wavelengths. Therefore, with proper photographic filtration the background noise due to the scattering of the light by the unwanted particles can be eliminated. Another advantage of using luminescent particles is the capability of effectively adding information regarding the flow direction on the trace. Gharib and Hernan [10] used the after glowing properties of optically activated phosphorescent particles to generate traces with variable intensity. The intensity decay on each trace was used to infer the flow direction.

With fluorescent particles, which are easier to make, one can obtain a similar effect by chopping the laser beam with a variable density filter. These filters, which are used as intensity modulators, can be designed to generate reference points to mark the beginning and ending of each trace. Gharib et.al. [11] used two small round openings at the two ends of the chopping filter's window to generate the reference points. A similar technique has been adopted by Agui and Jimenez [6].

In the chopping filter method a desired flow time scale could be resolved by controlling the illumination period through the rotation rate of the filter. The shape and intensity of the light pulse could only be changed by modifying the window's geometry and filter. In the latest version of the particle tracing technique developed by the authors, the chopping filter has been replaced by an acousto-optic device (Bragg cell). A single line laser beam diffracts to several frequency shifted beams as it goes through the Bragg cell. The frequency shifted beams are harmonics of the main beam which is also available after passing through the Bragg cell. The intensity distribution among the main beam and its harmonics is controlled by the RF voltage that is provided by a driver device. The intensity of the main beam can be modulated by an arbitrary wave function which is provided by an IBM AT to the driver device (Fig.1).

\section{IMAGE ACOUISITION AND DIGITIZATION}

Photographic recording of tracer images is still popular when high spatial resolution is required. The photographic recording time is normally controlled by the illumination period. In conventional modern particle tracing methods, either the photographic negative or positive is digitized by digitizing tables ([8], [9]) or video frame grabbers [10]. The digitizing process is controlled by a host computer. Commercial frame grabbers available for the $\mathrm{PC}$ type computers can digitize an image into a $512 \times 512$ pixel matrix with a 256 (8 bit) gray level resolution.

A real-time video recording system is desirable in order to eliminate the required development time associated with photographic negatives and to eliminate non-uniformity in the image signal-tonoise ratio, which is a function of the processing method. One major problem with commercial tube type or CCD (Charged Coupled Devices) based video systems is that their recording time is fixed at $1 / 30$ second (or 30 frames per second). Therefore, generating a streak which requires a continuous recording of the particle motion within a single frame is difficult if not impossible. To eliminate the photographic step, one needs to use CID (Charge Integrating Device) type cameras. The CID camera is the digital counterpart of photographic cameras. Its exposure time is controllable. Even though the exposure time may be varied from a few milli-seconds to several minutes, the updating framing rate is usually fixed at $1 / 30$ of a second. In the remainder of this section an integrated illumination and recording system based on the CID cameras will be described.

A CID camera (GE TN2250) with a 512 X 512 pixel resolution is synchronized with the Bragg cell through the IBM AT. At a command from the operator, the controller for the CID camera starts integrating the light signal received from the flow for a period of $\Delta \mathrm{T}_{1}$. At a later time, a specified light pulse similar to the one generated by the chopping filter starts illuminating the flow field with a period of $\Delta \mathrm{T}_{2}$ where $\Delta \mathrm{T}_{2}<\Delta \mathrm{T}_{1}$. The reference points are separated by a period of $\Delta \mathrm{T}$ which can be controlled independently. At the end of each $\Delta \mathrm{T}_{1}$ period, an image is sent to the IBM AT for digitization or to a video recorder (Sony 3/4" VCR) for a later digitizing process. The updating speed of the system is limited by the video rate which is $1 / 30$ of a second per image. See Figure 2 for the timing of different steps of synchronization. Figure 3 shows a sample streak image which was recorded using the CID camera in the configuration described above. A typical trace generated by the reference point method is shown in Figure 4. The trace ends with a tail which indicates the flow direction.

By removing the photographic steps, the quality of the digitized images has improved drastically. Therefore, any post-processing needed to remove noise has been eliminated.

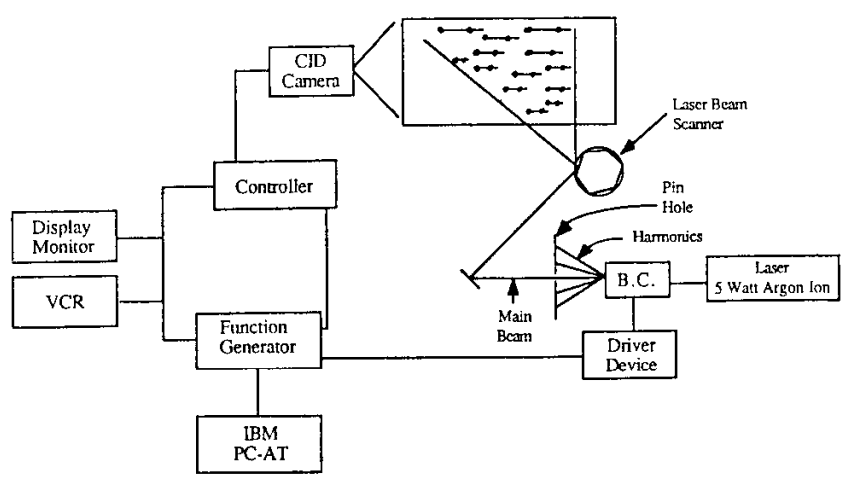

Figure 1: Schematic of the illumination and imaging system for the reference point technique. 


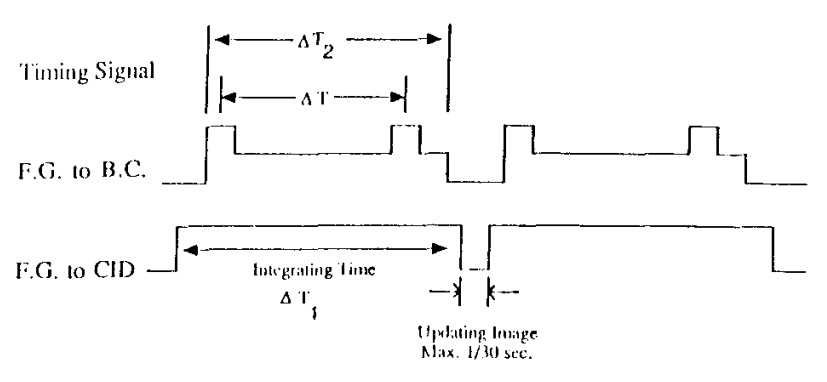

Figure 2: Timing diagram for different steps of the synchronization.

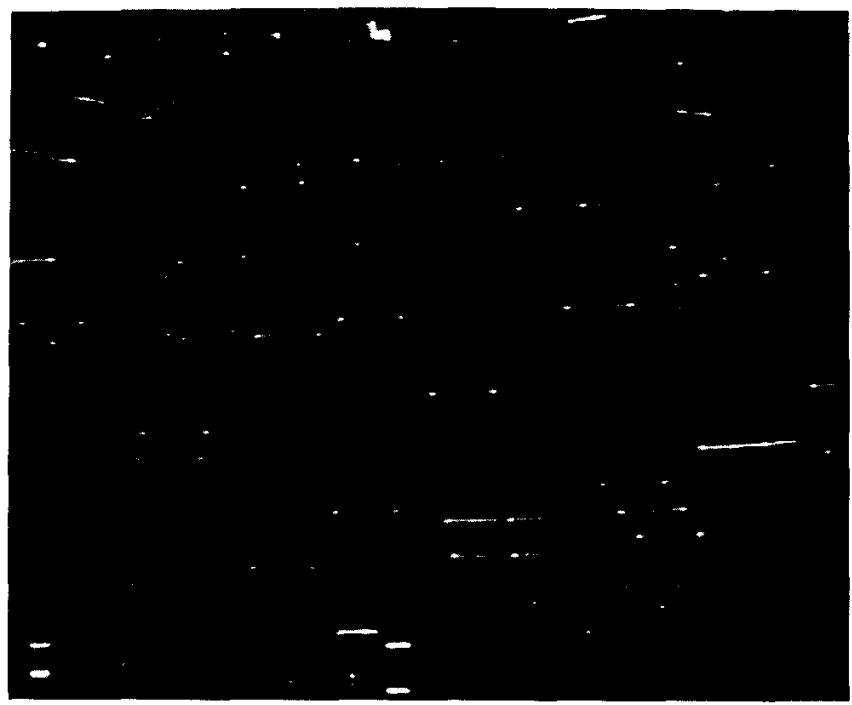

Figure 3: A typical streak image recorded with the charge integrating device (CID) camera.

\section{AUTOMATIC PARTICLE TRACING}

The next step in obtaining the velocity field is to educate the computer to recognize particle traces in the digital image. Automating this process has been the major challenge since the introduction of computers to the particle tracing technique. The methods that use digitizing tables and human operators to recognize traces and transform them to the digital form do not fall in the category of automatic particle tracing methods. These efforts suffer from the fact that recognizing process depends on the operator's judgement, who should bear in mind the flow direction and use some undefined criterion to identify the position and the length of each trace ([7] \& [8], among others).

The first attempt to automatically identify a contiguous group of data, representing traces from individual particles, was made by Dimotakis et.al. [9]. In their scheme a cluster of connected dots that had a predefined number of dots was recognized as a trace. A least squares parabolic fit was then computed for each separate group. The endpoints and length of each trace were saved for a subsequent interactive step to assign the local velocity vector and eliminate crossed or overlapping traces. This final stage of checking and restoring, which is interactive and time consuming in its nature, is common to many recent works that claim a certain degree of automation in their techniques (Altman, [12], Jonas \& Kent, [13], Elkins et.al,[14]).
The rest of this section is devoted to the description of the modified version of a fully automatic particle tracing technique that was originally developed and used by Gharib et.al. [11]. The essence of the technique is based on the assumption that the trace images generated by the particles contain the reference points and a tail to indicate the direction. In the present arrangement the digital images can be obtained from recorded images on VCR or directly from the camera. The software was designed to operate either on the displayed image on the monitor or on the stored information on the hard disk. The first step in the analysis of the digital images is to define the region of interest. By preexamination of a sample image, a gray level threshold is input to determine the minimum pixel value of what will be considered as a trace. The quality of the images make it possible to identify the trace regions with a single threshold value. The intensity distribution over a sample trace is presented in Figure 5. Once the threshold value is determined, it is applied to the remainder of the sequence of images. Using this threshold value, the processing program then sets any pixel value below the threshold level equal to zero (black) and saves the thresholded image.

In the next step, the program scans the image line by line to detect the first pixel greater than zero (or grey) of any potential trace region. To define a region, its perimeter of black pixels is found first by clockwise tracing around the region. The area on the interior of the perimeter is the trace region whose grayscale information and pixel coordinates stored in an array for further analysis (Figure 6). To eliminate any strongly curved traces, the program proceeds to calculate the centroid of the trace region. A centroid that does not lie on the perimeter or in the interior of the trace region indicates a curved trace leading to elimination of the entire trace region.

The next step is an automatic search inside the trace region for the reference points by scanning for pixel values greater than a computed peak-threshold. This peak-threshold is a function of the average gray level within the region and the standard deviation of this gray level average. The threshold value typically is greater than the average pixel value. Once the outline boundary of a reference region is defined in the same fashion that a trace region is defined, the centroid of it will be determined and assigned to the corresponding region (Figure 7). By counting the number of centroids in the region the program disqualifies any region with other than two reference points. Therefore, any region which was generated by an incomplete trace or by two crossed or overlapping traces is eliminated from further processing (Figure 8).

The length of the line that connects the reference points will be measured as the magnitude of the velocity vector and will be assigned to the halfway point between the two reference points (Figure 9). The flow direction is found by summing the distances from each reference point to all pixels of the trace region. The greater sum indicates the trailing end of the trace. Finally the velocity scalar, direction and mid-location of the trace is sorted in a file and the gray level value of the completed trace region is set to zero. Therefore, when the program continues the search for the next trace, it will not define the already detected trace region.

It is important to mention that the net time for the image recording from experiment, the digitization, the trace detection and the velocity vector field computation is approximately two minutes per image. We expect to optimize our software to reduce this 


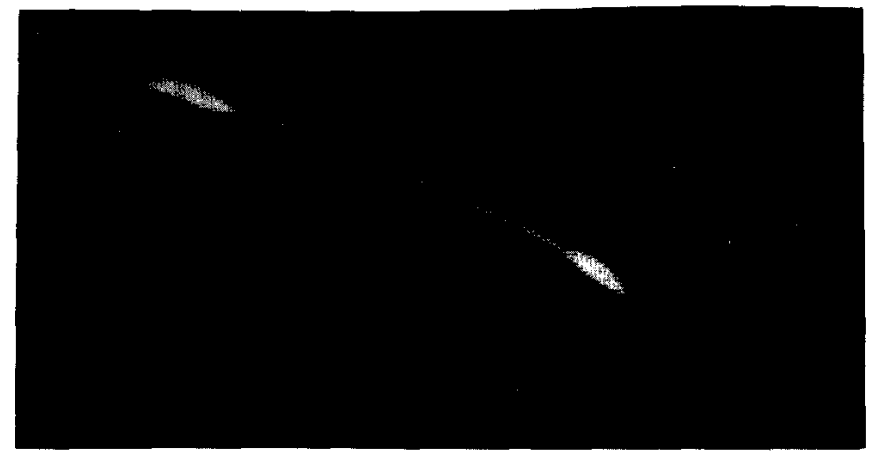

Figure 4: A typical trace generated by the reference point technique.

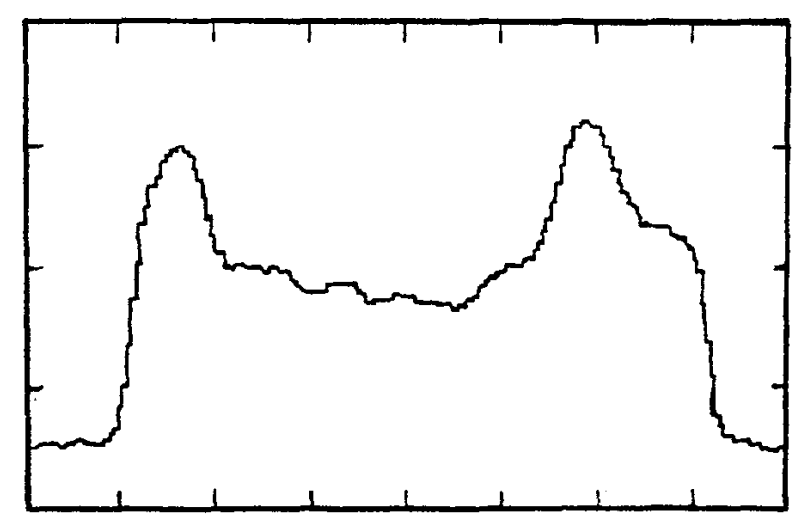

Figure 5: The intensity distribution for the trace in Figure 4.

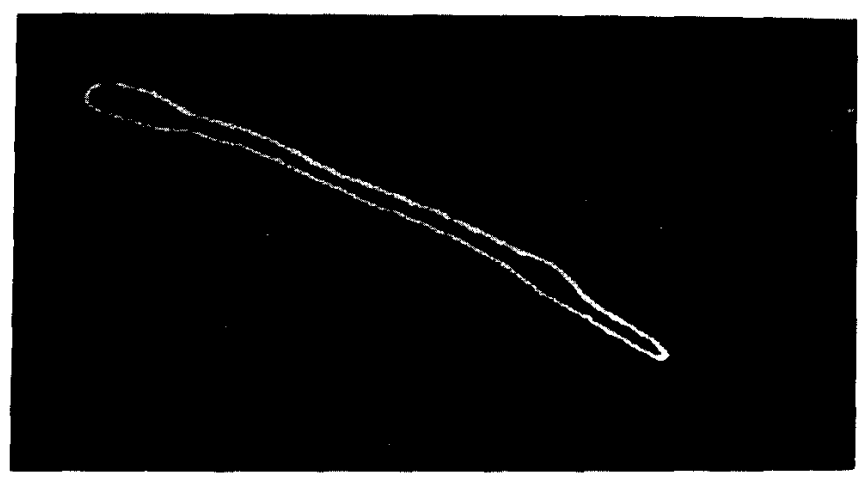

Figure 6: Boundary of the trace region.

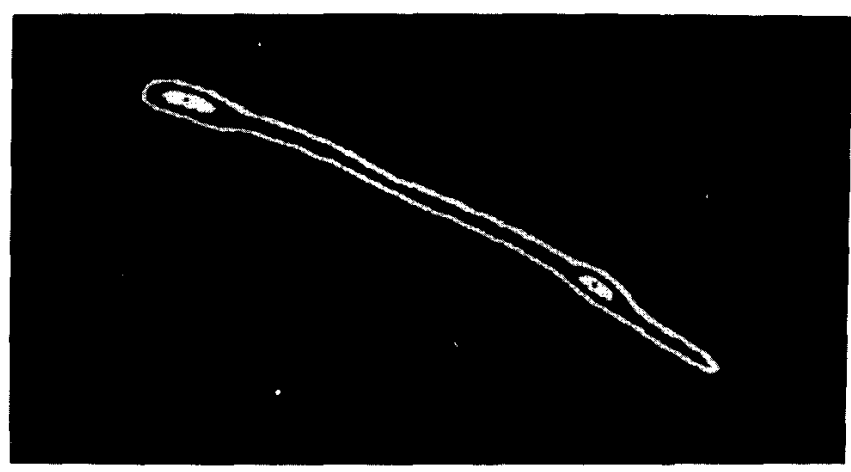

Figure 7: Reference point detection.

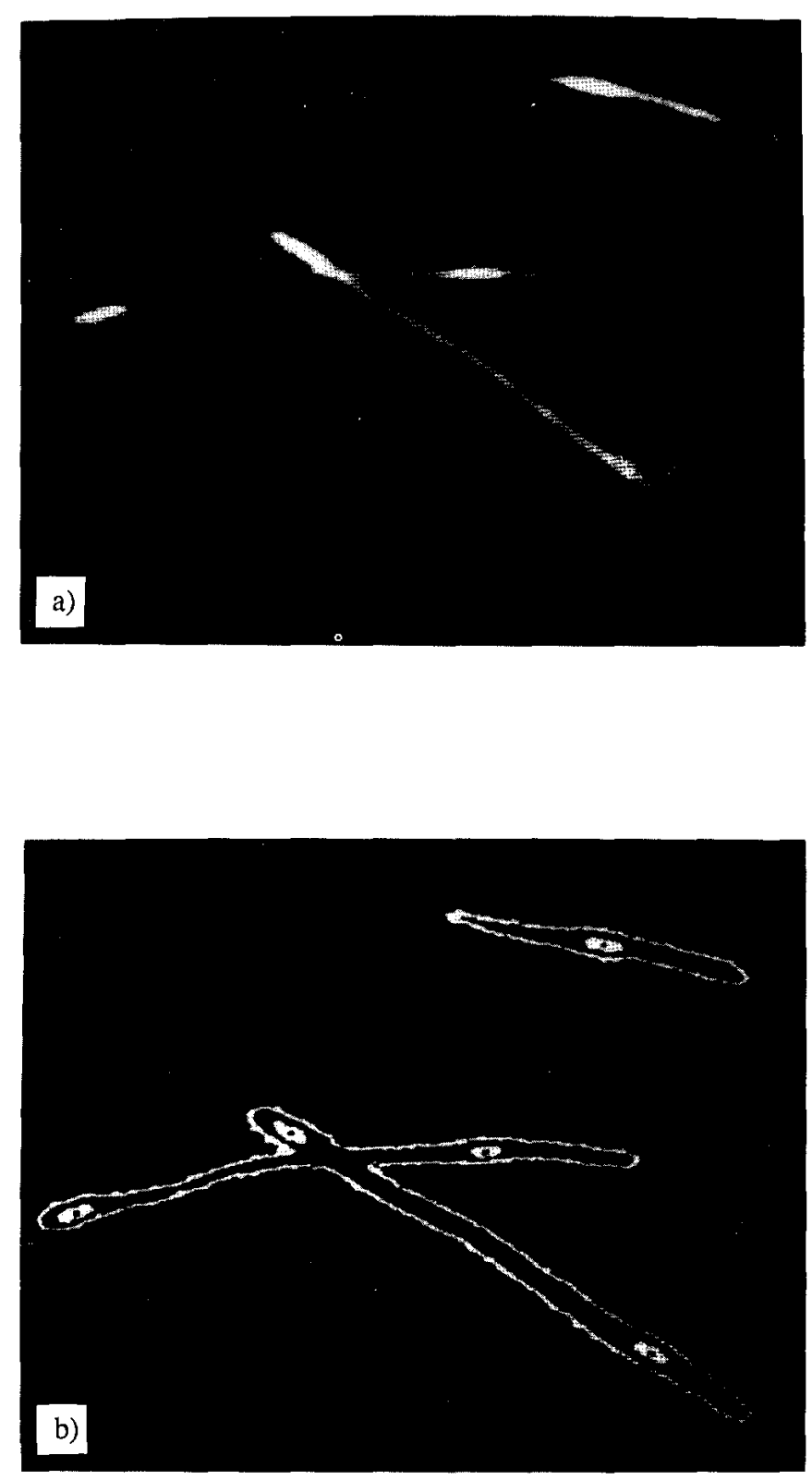

Figure 8: a) Original image. b) Processed Image

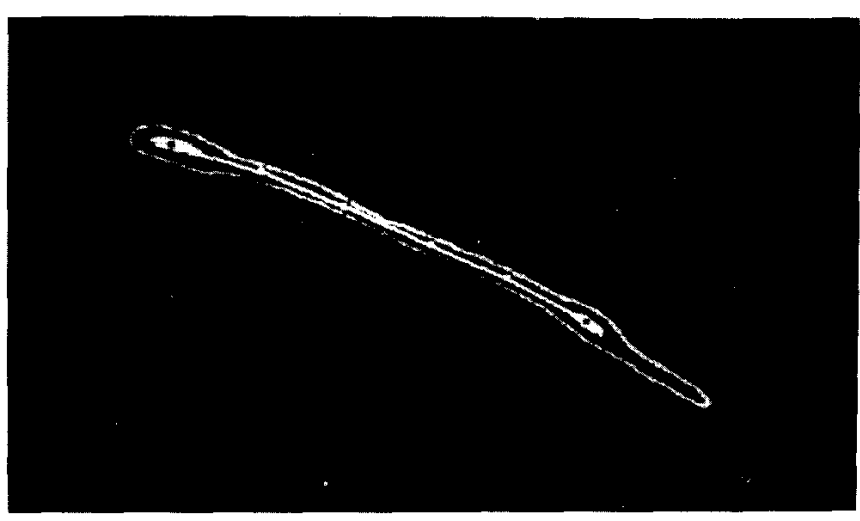

Figure 9: Constructed Velocity Vector 

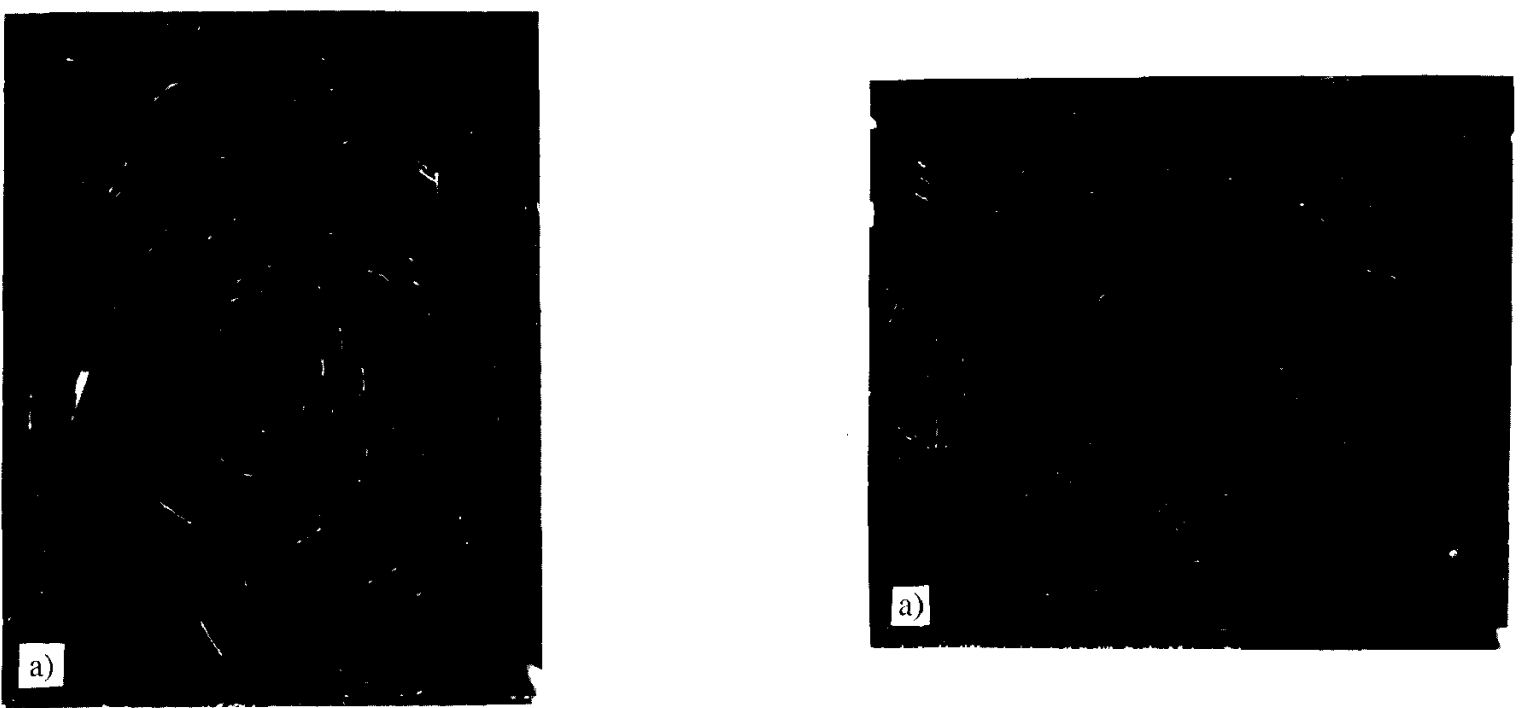

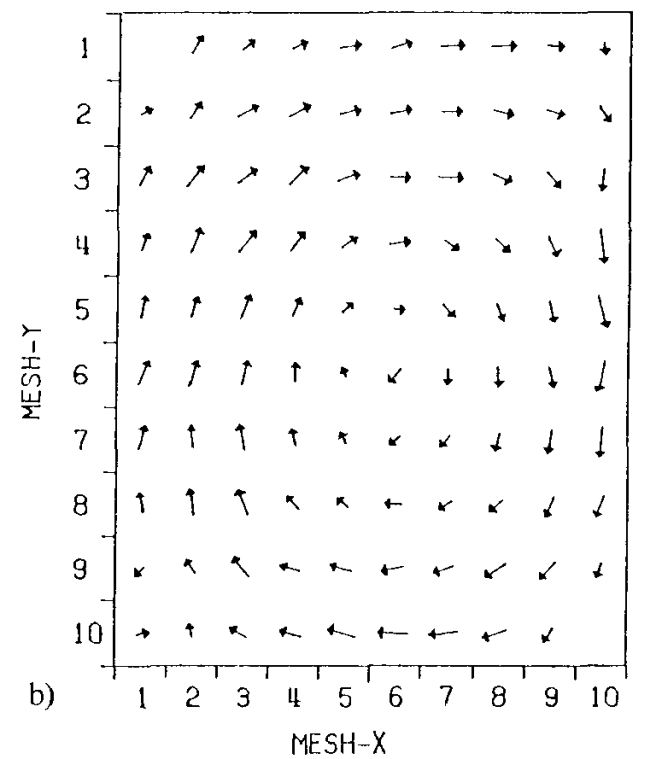

Figure 10: a) Sample flow field for $\mathrm{b} / \mathrm{h}=.8$.

b) Averaged velocity vector field.

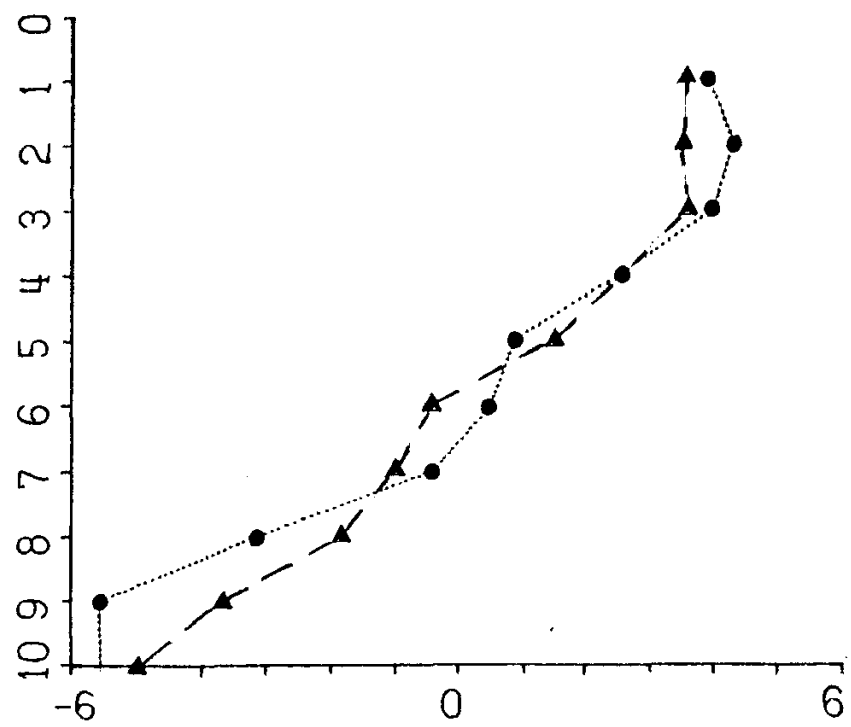

Figure 12: Comparison of particle tracing results $(\mathbf{\Lambda})$ to LDV measurements $(\bullet)$ for $\mathrm{b} / \mathrm{h}=.8$.

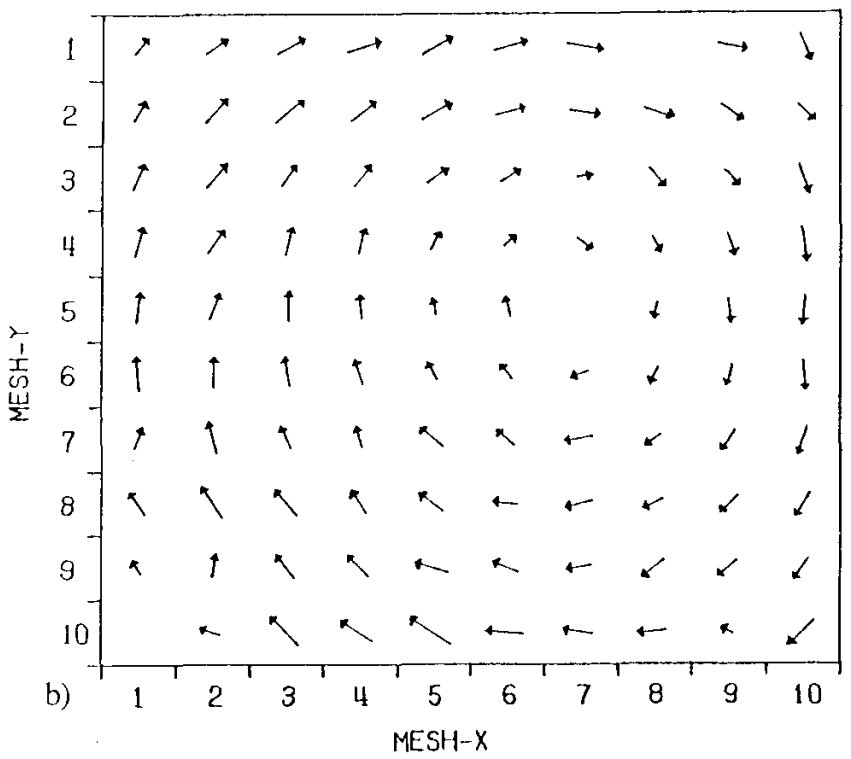

Figure 11: a) Sample flow field for $\mathrm{b} / \mathrm{h}=1.15$. b) Averaged velocity field.

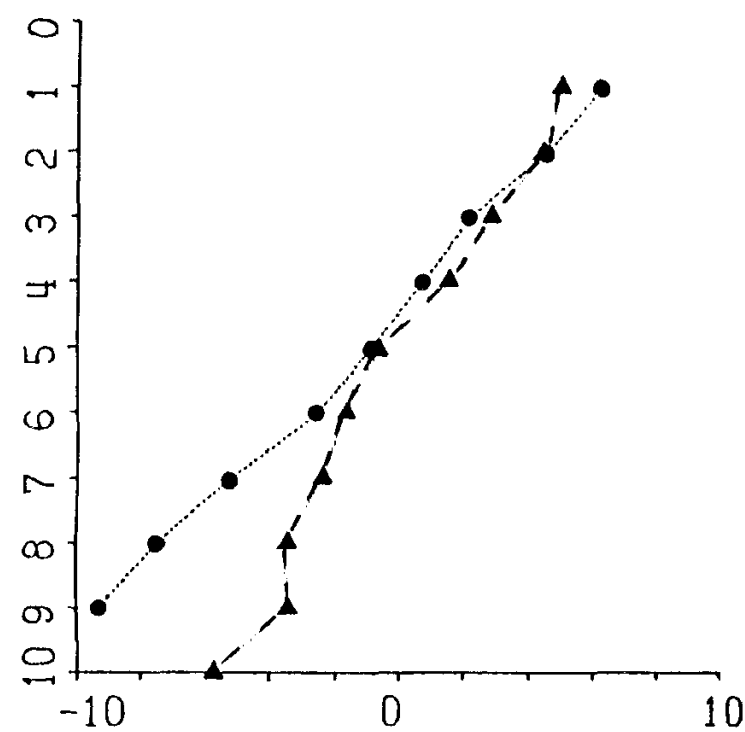

Figure 13: Same as Figure 12 for $b / h=1.15$. 
time to less than a minute per image. A typical reported time for obtaining similar information by other investigators (time for photographic processing not included) is on the order of one hour ([8], [9], \& [12], to mention a few).

In the next step a grid system is superimposed on the entire digitized field. The data for each trace is then read and assigned to the mesh square into which the mid-point of the trace falls. A vector summation of all the traces associated within a certain mesh square results in the mean velocity vector for the mesh square.

Gharib et.al. [11] applied the described technique to a recirculating flow inside a cavity with a width-to-depth ratio of .8. At this width-to-depth ratio, the flow inside the cavity is essentially a steady 2-dimensional recirculating stream. For a larger cavity width-to-depth ratio $(b / h>1)$, flow becomes unstable on a large scale and shows strong three-dimensional behavior. Figure 10 presents the trace images and vector field inside the cavity with $\mathrm{b} / \mathrm{h}=.8$ obtained from averaging 60 consecutive images.

Figure 11 shows a similar sequence for the $\mathrm{b} / \mathrm{h}=1.15$. To compare the Lagrangian velocity information to the information obtainable from single point Eulerian measurement techniques such as laser Doppler velocimetry, Gharib, et.al. [11] obtained one traverse of the velocity field at the middle of the cavity $(b / h$ $=.8$ ). Figure 12 shows an excellent agreement between this velocity profile and one obtained from the particle tracing method. Such agreement can be seen only on the upper half of the velocity profile for the large cavity configuration with $b / h=$ 1.15 (Figure 13). The disagreement between the two velocity profiles can be attributed to the strongly three-dimensional flow near the bottom of the cavity which drastically reduced the number of valid traces. Therefore, it was rarely possible to retrieve a sufficient number of particle streaks from even 60 realizations.

In another application of this technique we mapped the flow inside of a $7^{\circ}$ angle diffuser. Figure 14 presents the velocity profiles at three stations inside the diffuser. Each station presents average velocities at 20 to 30 grid points. Each grid point velocity presents an average of 30 velocity traces. It is interesting to mention that a mass conservation check at each station revealed an error of less than $5 \%$.

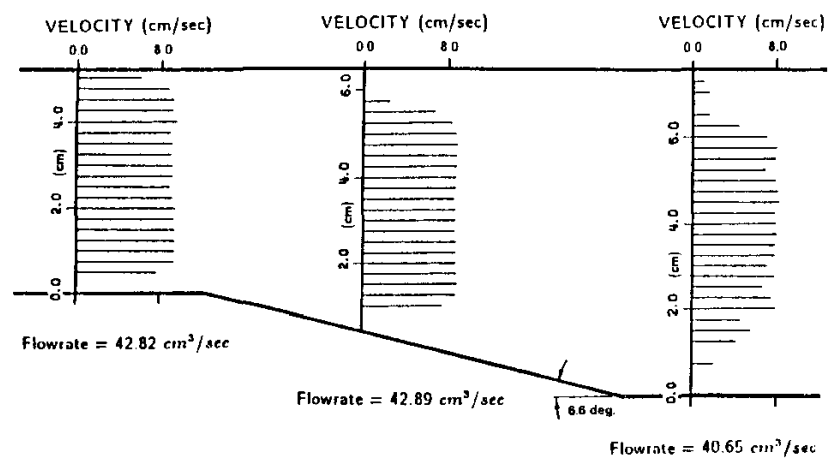

Figure 14: Velocity profiles at three stations inside a diffuser obtained by the reference point method.

\section{SOURCES OF MAJOR ERRORS}

The accuracy of the measurements and flow calculations in the described technique can be greatly influenced by several parameters. Optical and imaging errors which are common in most of the techniques that involve optical imaging have been discussed by several investigators ([15], [8]) and in some cases several techniques have been suggested to reduce the error [16]. In this section we will discuss some fundamental sources of errors that directly influence the flow computation and arise from imperfect conditions of the measurement technique. The magnitude of these errors, under certain circumstances, is by far larger than optical and imaging errors. In some cases, techniques that are developed to reduce these errors of measurement will be discussed.

\section{ERRORS OF COMPUTING EULERIAN VELOCITY FROM LAGRANGIAN MEASUREMENTS}

In the particle tracing technique we assume that the traces generated by the Lagrangian velocity of the particles are representing the Eulerian instantaneous velocity vector. However one needs to carefully consider the conditions that might violate the aforementioned assumption.

The trace generated by a particle is a result of the particle's Lagrangian motion. Therefore its length can be obtained by integrating its Lagrangian velocity

$$
\ell=\int_{0}^{\tau} \frac{\mathrm{d} \mathrm{s}}{\mathrm{dt}} \mathrm{dt}
$$

where $\underline{V}=\frac{d s}{d t} \underline{e_{t}}$,

$e_{t}$ is the tangential unit vector, and $s$ is the curvilinear coordinate. Following Altman [12], one can Taylor expand the Lagrangian velocity and integrate to obtain

$$
\begin{gathered}
\ell=\int_{0}^{\tau} \mathrm{V}(\mathrm{s}(\mathrm{t}), \mathrm{t}) \mathrm{dt} \\
\int_{0}^{\tau}\left(\mathrm{V}_{\mathrm{E}}(0,0)+\left.\frac{\partial \mathrm{V}}{\partial \mathrm{s}}\right|_{(0,0)} \ell+\left.\frac{\partial \mathrm{V}}{\partial \mathrm{t}}\right|_{(0,0)} \tau+\ldots\right) \mathrm{dt} \\
\quad \mathrm{V}_{\mathrm{E}}(0,0) \tau+\left.\frac{\partial \mathrm{V}}{\partial \mathrm{s}}\right|_{(0,0)} \tau \ell+\left.\frac{\partial \mathrm{V}}{\partial \mathrm{t}}\right|_{(0,0)} \tau^{2}+\text { H.O.T. }
\end{gathered}
$$

where $V_{E}$ is the Eulerian velocity and H.O.T. are higher order terms.

The measured velocity is usually obtained by dividing the trace length by exposure time,

$$
\mathrm{V}=\frac{\ell}{\tau}=\left.\mathrm{V}_{\mathrm{E}}\right|_{(0,0)}+\left.\frac{\partial \mathrm{V}}{\partial \mathrm{s}}\right|_{(0,0)} \ell+\left.\frac{\partial \mathrm{V}}{\partial \mathrm{t}}\right|_{(0,0)} \tau+\text { H.O.T. }
$$

Therefore the trace measurement will be a reasonable approximation of the Eulerian velocity if the velocity field changes slowly in space and time. If we assume that 
then

$$
\frac{\mathrm{dV}}{\mathrm{dt}}=\ddot{\mathrm{s}} \underline{\mathrm{e}_{\mathrm{t}}}-\frac{\dot{\mathrm{s}}^{2}}{\rho} \underline{e_{\mathrm{n}}}
$$

where $\rho$ is the principal radius of curvature and $e_{\mathrm{n}}$ is the normal unit vector. It is immediately apparent that trace curvature or an acceleration of the particle will have substantial contribution to the higher order terms. It is interesting to mention that large particle acceleration will cause a non-uniform intensity variation along the trace. Therefore one should avoid curved traces with non-uniform intensity for the purpose of velocity measurement.

\section{ERRORS DUE TO THE THREE-DIMENSIONAL MOTION OF THE PARTICLES}

As has been addressed by Gharib et.al [11] and Agui \& Jimenez [6], the most fundamental source of error in particle tracing methods is the erroneous trace length reading due to the lack of information regarding the entrance or exit of the particles from the light sheet. The nature of this error reveals no indication of its frequency of occurrence.

To understand the problem, let us define the traveling time of particle $\Delta \tau$ as the total time that the particle has traveled through the laser sheet during the illumination time period, $\Delta \mathrm{T}$. In conventional particle tracing methods, $\Delta \mathrm{T}$ corresponds to the camera's exposure time and the basic assumption is that $\Delta \mathrm{T}=\Delta \tau$ and particle velocity is usually defined as

$$
\mathrm{U}_{\mathrm{p}}=\frac{\Delta \mathrm{L}}{\Delta \mathrm{T}}
$$

where $\Delta \mathrm{L}$ is the photographically registered trace length. However, in a real situation, particles that enter the sheet after starting of the illumination or exit earlier than ending of the illumination period will have a traveling time shorter than $\Delta \mathrm{T}$. Usage of an improper $\Delta \mathrm{T}$ causes erroneous velocity readings as large as one hundred percent of the local mean velocity. This problem can be easily solved by the reference point technique because any particle that has remained in the sheet during the illumination period should have two bright reference points. Lack of one or both points on a trace disqualifies the trace for the velocity calculations.

In the cavity experiment with $\mathrm{b} / \mathrm{h}=1.5$ the disqualified particle traces were more than $60 \%$ of the total traces on each image. Other flow measurement techniques such as laser speckle or particle image displacement techniques (Adrian, [17]; Lourenco et.al., [18]) also suffer from the three-dimensional out-of-plane motion of the particles. However, only the methods that implement the reference point technique can accurately solve the problem.

\section{SAMPLING ERRORS}

The Nyquist sampling criterion suggests that the shortest resolvable wavelength in a flow image is twice as long as the average distance between traces. To avoid trace overlap problems, this average distance is usually set close to the average trace length in the image. Therefore features of the flow with a wavelength shorter than twice the average trace length will be undersampled. The resulting error in the velocity will be proportional to the integrated amplitude of the fluctuations that are filtered out by the undersampling. Agui and Jimenez [6] show that while this error might not affect the mean velocity measurements, it certainly contributes a major error to turbulent intensity measurements.

\section{ERROR IN FLOW COMPUTATION}

The trace detection scheme such as the one described in this paper that can accurately identify and reject traces due to overlapping and three-dimensional effects will severely reduce the number of traces per image. This results in a random distribution of traces over the image, and an interpolating scheme is required to reduce the irregularly spaced data to a regular rectangular grid. Several interpolation schemes are available where velocities at each mesh point are reconstructed by convolution with an adaptive Gaussian window [6] or a spline-thin-shell (Paihua de Montes, [19]) method over the entire velocity field.

To estimate the errors of interpolation Agui \& Jimenez [6] suggested an ingenious bootstrapping method where a data field composed of $\mathrm{N}$ velocity measurements interpolated onto a grid is successively resampled with $\mathbf{N}$ random samples. In this new sample some data from the original set are repeated while others are omitted. By comparing the velocities obtained through interpolating the new sample at the positions where trace velocities are known from an original data set, one can estimate the interpolation errors. The error estimates using the same bootstrapping technique is less reliable (mostly conservative) in the case of vorticity calculations. This is due to the fact that no $a$ priori knowledge of the vorticity field is available.

The Adaptive Gaussian Window (AGW) and thin-spline-shell (STS) techniques in combination with the bootstrapping method have been comprehensively implemented by Rignot and Spedding [20] and Spedding et.al. [21] to study the flow behind an unsteady flap. The velocity vector field which was obtained by the reference point technique is shown in Figure 15. Figures 16 and 17 show the interpolation velocity and vorticity fields, respectively, using the AGW and STS interpolating methods. Even though the reconstructed velocity fields are similar, isometric surfaces of the vorticity on the two grids clearly demonstrate how the vorticity distributions may be distorted by interpolation errors. Rignot \& Spedding concluded that one

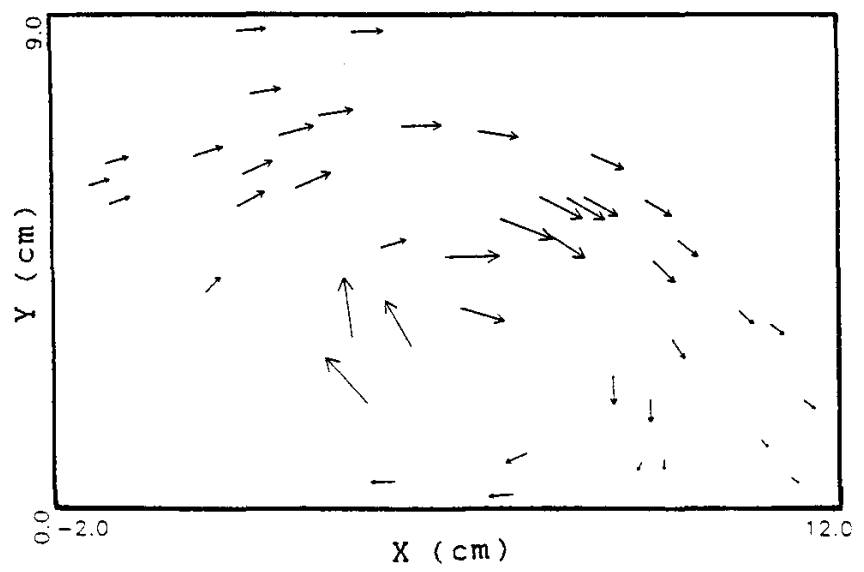

Figure 15: Velocity vector field behind an unsteady flap obtained by the reference point method. (Rignot and Spedding) 

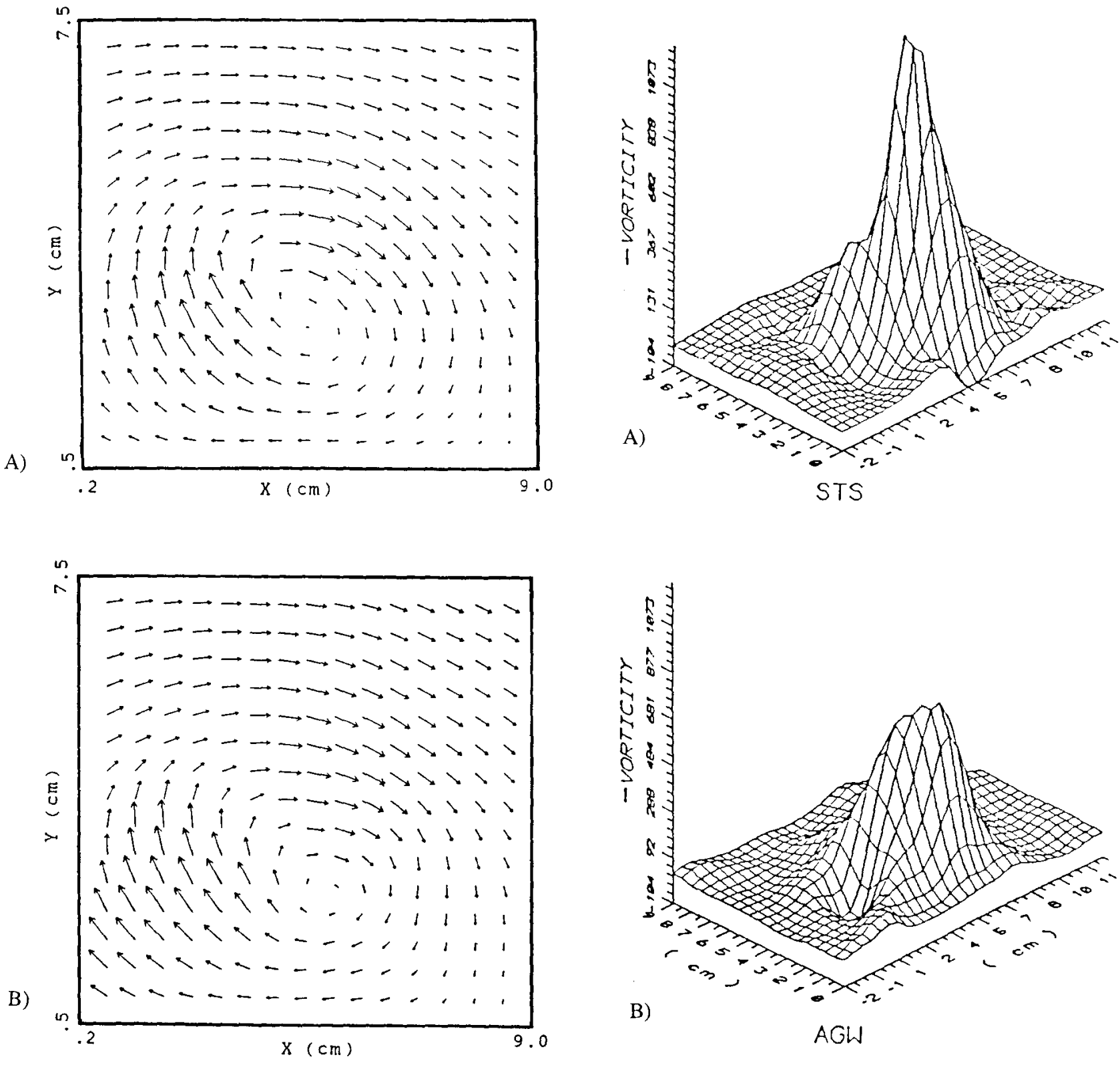

Figure 16: Interpolated velocity vector field for the flow in Figure 15.

A) STS method

B) AGW method

should make careful and informed decisions concerning the choice and implementation of interpolating techniques: the STS method would be the algorithm of choice where $\mathrm{N}$, the number of particles, is small. If original data has large cumulative errors, then AGW, being fast and easy to implement, may be a reasonable alternative.

\section{CONCLUSION}

A combination of the automatic particle tracing and one of the described interpolation methods can provide accurate and reliable velocity and vorticity measurements with an unprecedented speed. Currently, the speed of processing, which includes image acquisition and vector field construction, is one minute

per image. With the use of more powerful computers this time can be accelerated to the video rate ( 30 vector fields per second). The fact that fancier measurement methods such as laser speckle photography and speckle image displacement methods depend on the processing of photographic negatives makes the method described in this paper a candidate for becoming a real-time particle tracing technique. This is an important point when one considers the future uses of global velocity or vorticity information in real time active control of the various flow fields. A fundamental challenge of the future is to deal with the fast automatic construction of three-dimensional flow field with preferably a single camera. Methods that can simultaneously provide velocity vector and scalar fields such as temperature and concentration are in great demand. 


\section{ACKNOWLEDGEMENTS}

An initiation grant from the Engineering Foundation (GR Engng Fdn RI-A-86-7) has been instrumental in the development of the automatic particle tracing technique described in this paper. Currently a grant from DARPA/ACMP is supporting us in efforts to add the three-dimensional and multifield capability to the technique. Many colleagues and former students have helped us in bringing the technique to its present stage. We would like to thank Dr. Miguel Hernan for helping with the initial development of the idea, Barry Dyne and Oran Thomas for helping in the development of software, Thomas Phillips and Chee Yap for different stages of hardware development and Dr. Kathie Stuber for her careful laser doppler measurements.

\section{REFERENCES}

[1] Taneda, S. (1985), "Flow Visualization." Recent statistics on Turbulent Phenomena.

[2] Ahlborne, F. (1902), "On the Mechanism of Hydrodynamic Drag." (German) Abhandl Gebiete Naturwiss 17, Hamburg

[3] Prandtl, L. and O.G. Tietjens (1934), "Applied Hydroand Aeromechanics." McGraw-Hill, New York, 311 pp.

[4] Merzkirch, W. (1974), "Flow Visualization." Academic Press, New York, 250 pp.

[5] Saffman, P.G. (1965), "The Lift on a Small Sphere in a Slow Shear Flow." J. Fluid Mech. 22, 385-400.

[6] Agui, J.C. and J. Jimenez (1987), "On the Performance of Particle Tracking." J. Fluid Mech. 1985, 447-468.

[7] Wiese Nielson, K. Th. (1970), "Vortex Formation in Two-dimensional Periodic Wakes." Ph.D. Thesis, Oxford.

[8] Imaichi, K. and K. Ohmi (1983), "Numerical Processing of Flow-visualization Pictures-Measurement of Twodimensional Vortex Flow." J. Fluid Mech. 129, 283-311.

[9] Dimotakis, P.E., F.D. Debussy, and M.M. Koocheshahani (1981), "Particle Velocity Field Measurements in a Two-Dimensional Mining Layer." Phys. Fluids 24, 995-999.

[10] Gharib, M. and M. Hernan (1985), "Flow Velocity Measurement by Image Processing of Optically Activated Tracers," AIAA 85-0172-AIAA 23rd Aerospace Sciences Meeting, Reno, NV 1985.
[11] Gharib, M., B. Dyne, O. Thomas, and C. Yap (1986), "Flow Velocity Measurements by Image Processing of Optically Modulated Traces." Paper \#22, Proceeding of the AGARD Conference on Aerodynamic and Related Hydrodynamic Studies Using Water Facilities, Preprint \# 413.

[12] Altman, D.B. (1985), "Laboratory Studies of Internal Gravity Wave Critical Layers." Ph.D. Thesis, University of California, San Diego.

[13] Jonas, P.R. and P.M. Kent (1979), "Two-dimensional Velocity Measurement by Automatic Analysis if Trace Particles." Journal of Physics E. Scientific Instrumentation 12, 604-609.

[14] Elkins, R.E. III, G.R. Jackman, R.R. Johnson, E.R. Lindgren and J.K. Yoo (1977), "Evaluation of Stereoscopic Trace Particle Record of Turbulent Flow. Fields." Review of Scientific Instrumentation 48, 738-746.

[15] Wolf, P.R. (1983), "Elements of Photogrammetry, 2nd edition." McGraw-Hill, New York, 628 pp.

[16] Browand, F.K. and D.A. Plocher (1985), "Image Processing for Sediment Transport." Proceedings of the International Association for Hydraulic Research, 21 Congress, 1985.

[17] Adrian, R.J. (1984), "Scattering Particle Characteristics and their Effect on Pulsed Laser Measurements of Fluid Flow: Speckle Velocimetry vs. Particle Image Volumetry.", App. Opt. 23, 1690-1691.

[18] Lourenco, L., A. Krothapalli, J.M. Buchlin and M.L. Reighmuller (1986), "Noninvasive Experimental Technique for the Measurement of Unsteady Velocity Fields.", AIAA J. 24, 1715-1717

[19] Paihua de Montes, L. (1978), "Quelques Methodes Numeriques pour le Calcul de Fonctions Splines á une et Plusieurs Variables.", These de $3 e$ Cycle, Universite de Grenoble.

[20] Rignot, E. and G.R. Spedding (1987), "Performance Analysis of Automated Image Processing and Grid Interpolation Techniques for Fluid Flows.", USC Aerospace Engineering Internal Report.

[21] Spedding, G.R., T. Maxworthy and E. Rignot (1987), "Unsteady Vortex Flows over Delta Wings." Proc. 2nd AFOSR Workshop on Unsteady and Separated Flows, Colorado Springs, July 1987. 